\title{
Pregabalin in the management of partial epilepsy
}

This article was published in the following Dove Press journal:

Neuropsychiatric Disease and Treatment

29 July 2009

Number of times this article has been viewed

\section{Amir M Arain \\ Vanderbilt University Medical Center, Department of Neurology, Nashville, TN, USA}

\begin{abstract}
Pregabalin is a new antiepileptic medication that works by binding to alpha 2 delta subunit of the voltage-dependent calcium channels present in presynaptic neurons. Its pharmacokinetic advantages include rapid and almost complete absorption, lack of protein binding, linear kinetics, absence of enzyme induction, and absence of interactions with other drugs. Pregabalin was found effective as adjunctive therapy for refractory partial-onset seizures, with up to $51 \%$ responder at a dose of $600 \mathrm{mg} /$ day. The lowest effective dose was $150 \mathrm{mg} / \mathrm{day}$. Pregabalin is also approved for treatment of painful diabetic polyneuropathy, postherpetic neuralgia and pain with fibromyalgia. Studies also suggest a beneficial effect on sleep and generalized anxiety disorders. Its main adverse effects in randomized adjunctive trials in adults have been mild to moderate. Most common side effects were dizziness, ataxia, somnolence and diplopia. Weight gain was not prominent in pivotal pregabalin trials, but was more problematic in long-term postmarketing analyses in epilepsy patients. Pregabalin, with its potent antiseizure effect, favorable pharmacokinetic profile, and effectiveness in common co-morbidities is an important addition to the treatment of epilepsy.
\end{abstract}

Keywords: epilepsy, seizures, pregabalin, pharmacology, antiepileptic drugs, tolerability

Epilepsy is a common chronic neurological disorder that affects $0.5 \%$ to $1 \%$ of the population, ${ }^{1}$ and presents with recurrent unprovoked epileptic seizures. Surgical treatment may be curative in selected patients but overall there is no curative therapy for all epilepsy patients, and therapy is usually directed to prevention of seizures with antiepileptic drugs (AEDs). The management of epilepsy should be geared towards complete control of seizures while minimizing the occurrence of adverse events and improving the patient's quality of life. ${ }^{2}$ Approximately two thirds of patients become seizure-free with AED therapy, leaving the remaining third with persistent seizures. ${ }^{3}$ Patients may have to take AEDs for the rest of their life. Thus there is a need for newer and better-tolerated AEDs.

Before 1993 a limited number of AEDs were available. A variety of antiepileptic drugs have been introduced over the last 15 years. All of the newer AEDs have been shown to be effective as adjunctive agents in partial epilepsy. Marson et al's meta-analysis has shown that the response and tolerability of new AEDs in patients with refractory epilepsy are similar. Each drug was significantly better than placebo at preventing seizures. ${ }^{4}$

Pregabalin is the latest addition in the antiepileptic medication regimen in the United States. It was approved by Food and Drug Administration in 2005 as an add-on therapy for partial epilepsy, for painful diabetic polyneuropathy, postherpetic neuralgia, and fibromyalgia.
Correspondence: Amir M Arain A-0I I 8 Medical Center North, Nashville TN 37232, USA

$\mathrm{Tel}+\mathrm{I}(615)$ 936-259l

Fax +I (6I5) 936-0223

Email amir.arain@vanderbilt.edu 


\section{Mechanism of action}

Pregabalin was designed to be an analogue of GABA, but it does not act at GABA receptors. ${ }^{5}$ Pregabalin does not act on sodium channels, potassium channels, or glutamate receptors. Like gabapentin it works by binding to alpha 2 delta subunit of the P/Q-type voltage-sensitive $\mathrm{Ca}^{2+}$ channels (VSCC) which are present in presynaptic neurons. ${ }^{6,7}$ It does not block calcium channels rather it modulates calcium channels. ${ }^{6}$ In various animal models pregabalin exhibits anticonvulsant, anxiolytic and analgesic properties. ${ }^{5}$

\section{Pharmacology}

Pregabalin is rapidly and completely absorbed after oral dosing in the fasting state (bioavailability is $>90 \%$ ). Its absorption is not dose-dependent. Maximal plasma concentrations are reached in 1 hour after single or multiple doses, and steady state is achieved within 24 to 48 hours after repeated administration. Therefore, pregabalin can be taken with or without food. The pharmacokinetics of pregabalin is linear and predictable across the therapeutic dosage range (150 to $600 \mathrm{mg} /$ day). Pregabalin has a plasma half-life of 6.3 hours. It has to be given in 2 or 3 divided doses. It reaches steady-state plasma levels within 1 or 2 days of dosing.

Pregabalin is not protein bound and is excreted virtually unchanged by the kidneys. AEDs by the virtue of their protein binding and hepatic metabolism commonly results in important drug interactions that can have life-threatening clinical consequences. ${ }^{8}$ Pregabalin does not go through any significant hepatic metabolism. Pregabalin does not inhibit or induce hepatic enzymes. These properties enable it to cause no significant interactions with concomitant medications, eg, oral contraceptives. ${ }^{9,10}$ As was evident in a study of patients with refractory partial seizures in open-label, multiple-dose study with pregabalin $600 \mathrm{mg} /$ day, given as $200 \mathrm{mg} 3$ times a day for 7 days, in addition to maintenance monotherapy with valproate (VPA), phenytoin (PHT), lamotrigine (LTG), or carbamazepine (CBZ). The baseline antiepileptic medications were unaffected by concomitant pregabalin administration. ${ }^{11}$ However in another study pregabalin level decreased by $20 \%$ to $30 \%$ in the presence of enzyme-inducing antiepileptic drugs (eg, carbamazepine). ${ }^{12}$ Because pregabalin is excreted mostly unchanged through kidney, there is less need for therapeutic drug monitoring. However drug monitoring may be useful based on intra-individual comparisons of drug serum concentrations. ${ }^{13}$ Its excretion is proportional to creatinine clearance. In patients with renal insufficiency pregabalin dose has to be adjusted according to the creatinine clearance if lower than $60 \mathrm{~mL} / \mathrm{min}$. A 50\% reduction in pregabalin daily dose is recommended for patients with creatinine clearance between 30 and $60 \mathrm{~mL} / \mathrm{min}$. Pregabalin daily doses should be further reduced by approximately $50 \%$ for each additional 50\% decrease in creatinine clearance, as noted by Randinitis et al. ${ }^{14}$

\section{Pregabalin clinical trials}

Pregabalin's efficacy and safety have been studied in patient with partial epilepsy, with or without secondary generalization as an add-on therapy. Pregabalin was studied in 4 randomized, double-blind, placebo-controlled trials (Table 1).

French et al studied pregabalin's efficacy, safety, and tolerability in patients with partial seizures and to confirm the dose-response relationship. In this multicenter, doubleblind, randomized, placebo-controlled, parallel-group study, patients were given pregabalin in 2 divided doses over a 12-week double-blind phase. ${ }^{15}$

Patients with refractory partial seizures on 1 to 3 antiepileptic drugs were randomized to one of the five treatment groups (placebo or 50, 150, 300, and $600 \mathrm{mg}$ /day pregabalin, all administered twice daily). The intent-to-treat analysis included 453 patients. Seizure frequency reductions from baseline analyses showed $7 \%$ in the placebo group, $12 \%$ in the $50 \mathrm{mg} /$ day arm, 34\% in the $150 \mathrm{mg} /$ day arm, 44\% in the $300 \mathrm{mg} /$ day arm, and $54 \%$ in $600 \mathrm{mg} /$ day arm. ${ }^{15}$

Seizure responder rates $(50 \%$ or more reduction in seizure frequency) were $14 \%$ in placebo group, $15 \%$ in the $50 \mathrm{mg} /$ day arm, $31 \%$ in the $150 \mathrm{mg} /$ day arm, $40 \%$ in the $300 \mathrm{mg} /$ day arm and $51 \%$ in the $600 \mathrm{mg} /$ day arm. There was trend of better

Table I Summary of randomized, double-blind, placebo-controlled trials with pregabalin

\begin{tabular}{lllll}
\hline Author & Doses used (mg/day) & Seizure responder rate (\%) & Discontinuation rate (\%) & Most common side effects \\
\hline French $^{15}$ & $50,150,300,600$ in BID doses & $15,31,40,51$ & $7,1.2,14,24$ & Somnolence, dizziness \\
Arroyo $^{16}$ & 150,600 in TID doses & $14.1,43.5$ & $10,18.5$ & Dizziness, somnolence \\
Beydoun $^{17}$ & 600 in BID vs TID doses & 43,49 & 26,19 & Dizziness, somnolence \\
Elger $^{18}$ & 600 in BID vs I50-600 flexible dose & $45.3,31.3$ & 33,12 & Dizziness, ataxia \\
\hline
\end{tabular}

Abbreviations: BID, given in two divided doses; TID, given in three divided doses. 
efficacy with higher doses. The 150 to $600 \mathrm{mg}$ /day pregabalin groups were associated with greater reductions in seizures $(P \leq 0.0001)$ and greater responder rates compared with the placebo group $(P \leq 0.006)$. However the discontinuation rate due to side effects was also higher with higher dosages $(5 \%$ in placebo, $7 \%$ in the $50 \mathrm{mg}$ /day group, $1.2 \%$ in the $150 \mathrm{mg}$ /day group, $14 \%$ in the $300 \mathrm{mg} /$ day group, and $24 \%$ in the $600 \mathrm{mg}$ /day group). Most adverse events were of mild or moderate intensity, tended to occur sooner after initiation of pregabalin and resolved while patients remained on study treatment. Most common side effects were dizziness and somnolence.

Arroyo et al did a dose response study with pregabalin. In a multicenter 12-week, double-blind, randomized study in refractory partial epilepsy patients they evaluated pregabalin $150 \mathrm{mg}$ /day vs $600 \mathrm{mg} /$ day given in three divided dose. ${ }^{16}$ Pregabalin was significantly more effective than placebo in reducing the risk ratio ( $\mathrm{RR}$, a simple transformation of the seizure data and allows for the use of parametric statistics) in both $150 \mathrm{mg} /$ day and $600 \mathrm{mg} /$ day groups $(-11.5[P=0.0007]$ and $-31.4[P \leq 0.0001]$, respectively).${ }^{16}$

The RR values corresponded to seizure-frequency reductions from baseline of $1.8 \%, 20.6 \%$, and $47.8 \%$ for placebo, $150 \mathrm{mg} /$ day, and $600 \mathrm{mg} /$ day, respectively. They found that pregabalin efficacy was dose-related and more patients on pregabalin were responders compared to placebo $(P<0.0001)$. Responder rate for the $600 \mathrm{mg} /$ day pregabalin group was statistically superior to that of the $150 \mathrm{mg}$ /day pregabalin group $(P \leq 0.001)$.

Pregabalin was well tolerated in this study. Adverse events were mostly mild or moderate in intensity. Most common side effects reported were somnolence, dizziness, ataxia, diplopia, and weight gain. The median duration of any adverse events was similar among the $600 \mathrm{mg} /$ day pregabalin group (54 days) and the placebo group (55 days) and was shortest in the $150 \mathrm{mg}$ /day pregabalin group (28 days). The withdrawal rate because of adverse events was higher in the $600 \mathrm{mg}$ /day group (18.5\% at $600 \mathrm{mg} /$ day vs $10 \%$ at $150 \mathrm{mg} /$ day; $6.2 \%$ in the placebo group).

Beydoun et al studied pregabalin given in two vs. three divided doses in a multicenter, double-blind, randomized, parallel-group, placebo-controlled 12 weeks trial of refractory partial epilepsy patients. ${ }^{17}$ Pregabalin $600 \mathrm{mg}$ /day dose was administered in two or three divided doses. Pregabalin resulted in significant seizure frequency reductions compared to placebo $(P<0.0001)(53 \%$ for three times a day group vs $44 \%$ for twice daily group vs $1 \%$ increase for placebo). Fifty percent responder rates were $49 \%$ for three times a day group vs $43 \%$ for twice daily group compared with $9 \%$ for placebo $(P<0.001) .{ }^{17}$
The majority of side effects were mild to moderate in intensity in this study. Overall, dizziness was most frequently occurring side effects in all three treatment groups and it was dose dependent (38\% for three times a day and $42 \%$ for twice daily) than in placebo-treated patients (12\%). Other adverse events (AEs), eg, somnolence, ataxia, weight gain, amblyopia, asthenia, diplopia, and abnormal thinking occurred more frequently in patients randomized to the pregabalin groups. The withdrawal rate owing to adverse events was higher in the twice daily group than the three times a day group (26\% vs $19 \%) .{ }^{17}$

Elger et al studied pregabalin in fixed vs flexible dosing in randomized, double-blind, placebo-controlled 12 week study of patients with partial seizures refractory to AED treatment. ${ }^{18}$ Pregabalin was given either in fixed dose of $600 \mathrm{mg} /$ day given twice daily, or flexible dose of 150 to $600 \mathrm{mg}$ /day given twice daily. Pregabalin resulted in a reduction in seizure frequency of $35.4 \%$ for the pregabalin flexible-dose group, and $49.3 \%$ for the pregabalin fixed-dose group compared with $10.6 \%$ for the placebo group. ${ }^{18}$ Most adverse events were mild to moderate. Overall, dizziness was the most frequent side effect in all three treatment groups and was dose-dependent (5.3\% for the flexible dose group and 13.9\% for the fixed dose group; placebo $0 \%$ ). Other side effects seen more frequently in the pregabalin-treated group were ataxia, asthenia, somnolence, vertigo, and diplopia. The withdrawal rate owing to adverse events was higher in the fixed- than the flexible-dose group (32.8\% vs $12.2 \%) .{ }^{18}$

In all these four randomized placebo-controlled parallel trials of pregabalin as an add on therapy for partial epilepsy, the odds ratio for a $\geq 50 \%$ reduction in seizure frequency was 3.56 (CI 2.60 to 4.87 ) for pregabalin (all doses pooled) relative to placebo. ${ }^{19}$

The long-term efficacy of pregabalin as add on therapy in partial epilepsy was studied in four long term open label extension studies of 1480 patients. Majority of patients received pregabalin $\geq 450 \mathrm{mg}$ /day. Over the last 6 and 12 months of pregabalin across these studies, proportion of seizure freedom ranged from $7.4 \%$ to $24.2 \%$ and from $4.5 \%$ to $18.4 \%$. Seizure responder rates were $41 \%$ to $60 \%$ during the subsequent 6,12 , or 24 months..$^{20,21}$

\section{Postmarketing epilepsy studies}

Since the marketing of pregabalin a number of published open-label and placebo controlled studies have confirmed the efficacy of pregabalin. Selected studies are presented in relation to specific aspects of pregabalin.

In a placebo-controlled randomized study of pregabalin in refractory epilepsy, patients were randomized to adjunctive 
pregabalin fixed dose (600 mg/day twice daily), or pregabalin flexible dose (150 and $300 \mathrm{mg} /$ day for 2 weeks each; 450 and $600 \mathrm{mg} /$ day for 4 weeks each, twice daily) or placebo for 12 weeks. ${ }^{22}$ Dosage was adjusted based on tolerability and maintained when a 4-week seizure-free period was achieved. Both pregabalin regimens significantly reduced seizure frequency compared with placebo, by $35.4 \%$, for flexible dose $(P=0.0091)$ and $49.3 \%$ for fixed dose $(P=0.0001)$ vs $10.6 \%$ for placebo, and the fixed-dose group was superior to the flexible-dose group $(P=0.0337)$. Most adverse events were mild or moderate and incidence of adverse events and discontinuations were lower in patients when dosing was individualized to optimize efficacy and tolerability. Discontinuation rates due to adverse events were higher in fixed dose group and discontinuation was earlier than other groups $\left(32.8 \%\right.$ fixed vs $12.2 \%$ flexible group. ${ }^{22}$

In another trial evaluating cost effectiveness of pregabalin to other antiepileptic medications, the cost-effectiveness of pregabalin therapy in terms of cost per seizure-free day gained or cost per quality-adjusted life-years gained compares favorably with published estimates for other add-on antiepileptic medications. ${ }^{23}$

In analysis of pooled patient data from four short-term placebo studies of pregabalin (150 to $600 \mathrm{mg}$ /day) demonstrates that pregabalin is an effective and well tolerated addon treatment for partial seizure with or without secondary generalization. ${ }^{24}$ Weight gain, reported by $5.4 \%$ to $17.1 \%$ of patients across pregabalin dosing groups, was dose-related (highest in the $600 \mathrm{mg} /$ day group) and resulted in withdrawal from study in only $0.74 \%(6 / 810,4$ of whom were in $600 \mathrm{mg}$ /day group) pregabalin-treated subjects. Weight gain reached steady state in 85 days. $^{24}$

Weight gain has been a significant adverse effect with pregabalin. In another study evaluating the utility of short counseling program to prevent weight gain, showed the mean bodyweight increase, compared to baseline, of $2.5 \mathrm{~kg}$ ( $\mathrm{SD} \pm 3.7 \mathrm{~kg}$; median: $2.0 \mathrm{~kg}$ ) and $4.0 \mathrm{~kg}(\mathrm{SD} \pm 4.1 \mathrm{~kg}$; median: $4.0 \mathrm{~kg} ; \mathrm{N}=60$ ) at the 3 - and 6-month follow-up, respectively. ${ }^{25}$ The body mass index increment was 0.9 ( $\mathrm{SD} \pm 1.3$; median: 0.6 ) and 1.4 (SD \pm 1.4 ; median: 1.3 ) for the 3- and 6-month follow-up, respectively. ${ }^{25}$

\section{Pregabalin in epilepsy patients with intellectual and developmental disability}

Epilepsy is common in patients with intellectual and developmental disability (I/DD) and they tend to have medically refractory epilepsy, often with multiple seizure types. ${ }^{26}$ Pregabalin efficacy and tolerability has been studied in institutionalized patients with epilepsy and intellectual and developmental disability (I/DD) mostly in a retrospective study design. ${ }^{27,28}$ Huber et al retrospective study of pregabalin in 32 institutionalized patients with therapy-resistant epilepsy and intellectual disability showed modest efficacy. ${ }^{28}$ The retention rate was $75 \%$ after 6 months. Six patients $(18.75 \%)$ were responders (50\% seizure reduction). No patient was seizure free. Eight patients had side effects that were essentially impairing. Weight gain, somnolence, asthenia, and ataxia were the most frequent adverse effects. Rare adverse events were severe mental slowing and loss of daily life capacities on a low dose of pregabalin in one patient and increase in auto-aggression in another patient. After 12 months, the retention rate dropped to $40.6 \%$, the responder rate was $25 \%$, and one patient was seizure free.

Modur et al studied efficacy and tolerability of pregabalin in a retrospective study of seven institutionalized patients with I/DD. ${ }^{27}$ The primary efficacy measure was the change in the median frequency of seizure days per week between the baseline ( 8 weeks prior to initiating pregabalin) and treatment (12 weeks of titration and maintenance) periods. The mean dose of pregabalin was $293 \mathrm{mg}$ /day (range 150 to $350 \mathrm{mg} /$ day). Pregabalin was efficacious, resulting in a significant reduction in the median frequency of seizure days/week between baseline and treatment ( 1.38 vs 0.50 , $P=0.018$ ). The $50 \%$ responder rate was $71 \%$. The adverse effects at last follow-up (mean 13 months) included weight gain, myoclonus, and sedation. Though Modur et al study had a smaller sample size but their responder rate was better than Huber study. The reason for the discrepancy may be that these studies had different dosages - mean dose $390 \mathrm{mg} /$ day in Huber vs 293 mg/day in Modur. Modur et al studied efficacy at the end of 12 weeks of treatment while Huber evaluated efficacy at 6 months and 12 months of treatment; thus Huber et al had a longer study duration that may account for the relatively low responder rate.

In a postmarketing retrospective analysis, efficacy and tolerability of pregabalin was studied over 15 months, in 119 patients from two epilepsy clinics. Forty-six (38.6\%) patients had discontinued pregabalin, 33\% (15/46) of patients discontinued pregabalin for lack of efficacy, while $66 \%$ stopped pregabalin for adverse effects. ${ }^{29}$ The most common adverse effect causing discontinuation was behavioral and psychiatric adverse effects, including agitation, irritability, and depression (9 patients $-20 \%$ of those discontinuing). Weight gain was the second most common reason for 
discontinuation ( 7 patients, $15 \%$ ). The average weight change was $+12 \mathrm{lb}(5.5 \mathrm{~kg})$ (range -8 to $+71 \mathrm{lb} ; 3.6$ to $32.3 \mathrm{~kg})$ and average weight change per month was $1.1 \mathrm{lb}(0.5 \mathrm{~kg}){ }^{29}$ Twenty-nine (33\%) patients had a $>10 \%$ increase in weight. The median reduction in seizure frequency was $41 \%$. Pregabalin efficacy postmarketing was similar to that in clinical trials. However, behavioral adverse effects and weight gain were the most important reasons for discontinuation.

Pregabalin is the latest addition to the armamentarium against epilepsy. Its pharmacological and pharmacokinetic properties make it favorable for its use in clinical practice. Pregabalin's potent anticonvulsant activity (seizure responder rate of $51 \%$ ) is helpful in its clinical use. In clinical trials pregabalin has been found to be highly effective and well tolerated as adjunctive therapy for the treatment of patients with partial onset seizures with or without secondary generalization. Pregabalin's predictable oral absorption, lack of interaction, lack of protein binding, lack of hepatic metabolism, and linear pharmacokinetics are favorable. However weight gain, behavioral adverse effects, and myoclonus have to be carefully monitored by the clinicians while using pregabalin.

\section{Effect on sleep in epilepsy patients}

Sleep problems are a common issue with epilepsy patients and lack of sleep can worsen seizures. Pregabalin has been reported to enhance NREM sleep and reduces REM sleep in rats. ${ }^{30}$ Similarly in humans it improves sleep quality. In a trial of adult patients with partial epilepsy treated with pregabalin underwent 24-hour ambulatory polysomnography. Sleep quality was assessed with Epworth Sleepiness Scale (ESS) before and after 3 months treatment with pregabalin. ${ }^{31}$ A significant increase in REM sleep and a decrease of stage 2 NREM sleep was noted with pregabalin use. Epworth Sleepiness Scale showed a significant increase in the score but did not reach the pathological cut-off value (mean ESS score $<10$ ). The increase in REM sleep may indicate an improvement in nocturnal sleep quality while the increase of ESS score indicates that daytime somnolence is a minor adverse effect of prebalin. ${ }^{31}$

\section{Pregabalin in other neurological/ psychiatric disorders}

Comorbidities are important considerations in epilepsy patients in choosing the AED. AEDs are now often used to treat other disorders besides epilepsy and their efficacy is established in some non-epilepsy disorders both in neurology and psychiatry. ${ }^{32}$ In a randomized controlled 5-week trial of pregabalin in diabetic peripheral neuropathic pain, patients on doses of 300 or $600 \mathrm{mg} /$ day showed significant improvements in endpoint mean pain score vs placebo $(P=0.0001) .{ }^{33}$ Sustained improvements in pain and sleep quality were seen as early as week 1. A dose-related response was seen as a $50 \%$ reduction in pain compared to baseline at $46 \%$ in the $300 \mathrm{mg} /$ day arm and 48\% in the $600 \mathrm{mg}$ /day arm. Pregabalin was well tolerated with a low discontinuation rate. ${ }^{33}$ This trial showed pregabalin's efficacy in the treatment of diabetic peripheral neuropathic pain.

In another randomized controlled 8-week trial of pregabalin in the treatment of postherpetic neuralgia, pregabalintreated patients had significantly greater decreases in pain score than placebo (3.60 vs 5.29, $P=0.0001) .{ }^{34}$ Pain was significantly reduced very early in the trial. Similar to the trial in diabetic peripheral neuropathy, sleep also improved in patients treated with pregabalin compared to placebo $(P=0.0001)$. The side effects were generally mild to moderate. ${ }^{34}$

More recently pregabalin was studied in fibromyalgia in a randomized, controlled 13-week trial. ${ }^{35}$ Patients in all pregabalin groups (300, 450 and $600 \mathrm{mg}$ /day) showed statistically significant improvement in endpoint mean pain score. Similarly, all patients showed statistically significant improvement in assessments of sleep. ${ }^{35}$ Dizziness and somnolence were the most frequently reported adverse events.

Pregabalin has been evaluated for generalized anxiety disorder in 4 placebo-controlled clinical trials. Patients were randomized to 6 weeks of double-blind treatment with pregabalin 400 or $600 \mathrm{mg} /$ day, venlafaxine $75 \mathrm{mg} /$ day, or placebo. ${ }^{36}$ Pregabalin showed significantly greater improvement in anxiety score even at week 1. Pregabalin was concluded to be safe, well tolerated, and rapidly efficacious across the physical-somatic as well as the emotional symptoms of anxiety in primary care and psychiatric settings. Pregabalin has been approved in the European Union for generalized anxiety disorder.

\section{Pregabalin tolerability}

Pregabalin was found to be very well tolerated in the initial placebo-controlled adjunctive trials in partial epilepsy. The treatment-related adverse events were mild to moderate, appeared in the beginning of treatment, and resolved while patients remained on study treatment. Most common side effects were dizziness, ataxia, somnolence, and diplopia. Weight gain and behavioral side effects were not prominent in pivotal pregabalin trials. However in postmarketing analysis of pregabalin in epilepsy patients, the most common adverse effects causing discontinuation were behavioral 
adverse effects (agitation, irritability, and depression) and weight gain. Of note, no systemic side effects have been seen with pregabalin but myoclonus has been reported in 4 patients who developed new onset myoclonus at a dose of 50 to $600 \mathrm{mg} /$ day. ${ }^{37}$ Patients had focal or multifocal myoclonus without any EEG changes. Myoclonic jerks significantly decreased after dose reduction of pregabalin. ${ }^{37}$

\section{Place of pregabalin in epilpesy therapy}

Pregabalin is effective in partial epilepsy, but its efficacy in generalized epilepsy is not established. It should be considered as an early adjunctive therapy in partial epilepsy because of its ease of use, linear kinetics and lack of drug-drug interactions. ${ }^{19-21,24,38}$ Pregabalin's lack of drug-drug interaction makes it a particularly good choice in elderly patients who commonly are on multiple medications. In patients with hepatic insufficiency pregabalin along with levetiracetam and gabapentin are a good choice because of the absence of hepatic metabolism. Pregabalin's efficacy in multiple conditions makes it an earlier choice in epilepsy patients who suffer from such conditions. Since anxiety is a common comorbidity in epilepsy patients, ${ }^{39}$ patients with epilepsy and anxiety may benefit from pregabalin. Similarly in epilepsy patients with diabetic polyneuropathy and fibromyalgia, pregabalin would be a very appropriate add-on therapy.

Because of the potential side effect of weight gain patients should be counseled about routine exercise and their weight should be closely monitored when they are started on pregabalin. Similarly patients started on pregabalin should also be monitored for behavioral adverse effects, especially in epilepsy patients with intellectual and developmental disability.

The prescribing information recommends an adult starting dose of $50 \mathrm{mg}$ three times a day or $75 \mathrm{mg}$ twice a day, with subsequent escalation by 100 to $150 \mathrm{mg}$ every week up to a maximum of $300 \mathrm{mg}$ twice daily. These recommendations are based on the dose used in pivotal trials. However because of clinical experience and evidence from postmarketing analyses, the author favors a lower starting dose of $50 \mathrm{mg}$ twice daily or $75 \mathrm{mg}$ at bedtime because of excessive sedation especially in elderly patients, with escalation to $75 \mathrm{mg}$ twice daily after 1 week if the starting dose is well tolerated. In patients at higher risk for behavioral-psychiatric adverse effects, the starting dose can even be smaller, at $50 \mathrm{mg}$ at bedtime. In patients who tolerate pregabalin but still have uncontrolled seizures on $300 \mathrm{mg}$ twice a day, the author recommends splitting the dose to $200 \mathrm{mg}$ three times a day for better efficacy. However three times a day dosing may decrease compliance and hence benefits and risks have to be weighed.

Overall pregabalin is a good addition to the armamentarium against epilepsy. Its reliable absorption, linear kinetics, better efficacy, lack of drug-drug interaction, and lack of hepatic metabolism make it an attractive adjunctive therapy in controlling seizures. Pregabalin monotherapy trials will be needed to evaluate it as first-line therapy in new onset epilepsy or for conversion monotherapy in less refractory partial epilepsy.

\section{Disclosures}

The author has received research grant support from Pfizer and has given occasional lectures sponsored by the Pfizer speakers bureau.

\section{References}

1. Sander JW, Shorvon SD. Epidemiology of the epilepsies. J Neurol Neurosurg Psychiatry. 1996;61(5):433-443.

2. Arain A. Medical therapy of epilepsy. Expert Opin Ther Pat. 2007;17(8):955-964.

3. Kwan P, Brodie MJ. Early identification of refractory epilepsy. N Engl J Med. 2000;342(5):314-319.

4. Marson AG, Kadir ZA, Chadwick DW. New antiepileptic drugs: a systematic review of their efficacy and tolerability. $B M J$. 1996;313(7066):1169-1174.

5. Vartanian MG, Radulovic LL, Kinsora JJ, et al. Activity profile of pregabalin in rodent models of epilepsy and ataxia. Epilepsy Res. 2006;68(3):189-205.

6. Taylor CP, Angelotti T, Fauman E. Pharmacology and mechanism of action of pregabalin: the calcium channel alpha2-delta (alpha2-delta) subunit as a target for antiepileptic drug discovery. Epilepsy Res. 2007;73(2):137-150.

7. Dooley DJ, Donovan CM, Meder WP, Whetzel SZ. Preferential action of gabapentin and pregabalin at $\mathrm{P} / \mathrm{Q}$-type voltage-sensitive calcium channels: inhibition of $\mathrm{K}+$-evoked [3H]-norepinephrine release from rat neocortical slices. Synapse. 2002;45(3):171-190.

8. Patsalos PN, Perucca E. Clinically important drug interactions in epilepsy: interactions between antiepileptic drugs and other drugs. Lancet Neurol. 2003;2(8):473-481.

9. Harden CL, Leppik I. Optimizing therapy of seizures in women who use oral contraceptives. Neurology. 2006;67(12 Suppl 4):S56-S58.

10. Perucca E. Clinically relevant drug interactions with antiepileptic drugs. Br J Clin Pharmacol. 2006;61(3):246-255.

11. Brodie MJ, Wilson EA, Wesche DL, et al. Pregabalin drug interaction studies: lack of effect on the pharmacokinetics of carbamazepine, phenytoin, lamotrigine, and valproate in patients with partial epilepsy. Epilepsia. 2005;46(9):1407-1413.

12. May TW, Rambeck B, Neb R, Jurgens U. Serum concentrations of pregabalin in patients with epilepsy: the influence of dose, age, and comedication. Ther Drug Monit. 2007;29(6):789-794.

13. Johannessen SI, Tomson T. Pharmacokinetic variability of newer antiepileptic drugs: when is monitoring needed? Clin Pharmacokinet. 2006;45(11):1061-1075.

14. Randinitis EJ, Posvar EL, Alvey CW, Sedman AJ, Cook JA, Bockbrader HN. Pharmacokinetics of pregabalin in subjects with various degrees of renal function. J Clin Pharmacol. 2003;43(3):277-283.

15. French JA, Kugler AR, Robbins JL, Knapp LE, Garofalo EA. Doseresponse trial of pregabalin adjunctive therapy in patients with partial seizures. Neurology. 2003;60(10):1631-1637. 
16. Arroyo S, Anhut H, Kugler AR, et al. Pregabalin add-on treatment: a randomized, double-blind, placebo-controlled, dose-response study in adults with partial seizures. Epilepsia. 2004;45(1):20-27.

17. Beydoun A, Uthman BM, Kugler AR, Greiner MJ, Knapp LE, Garofalo EA. Safety and efficacy of two pregabalin regimens for add-on treatment of partial epilepsy. Neurology. 2005;64(3):475-480.

18. Elger CE, Brodie MJ, Anhut H, Lee CM, Barrett JA. Pregabalin add-on treatment in patients with partial seizures: a novel evaluation of flexibledose and fixed-dose treatment in a double-blind, placebo-controlled study. Epilepsia. 2005;46(12):1926-1936.

19. Lozsadi D, Hemming K, Marson AG. Pregabalin add-on for drugresistant partial epilepsy. Cochrane Database Syst Rev. 2008(1): CD005612.

20. Ryvlin P, Perucca E, Rheims S. Pregabalin for the management of partial epilepsy. Neuropsychiatr Dis Treat. 2008;4(6):1211-1224.

21. Warner G, Figgitt DP. Pregabalin: as adjunctive treatment of partial seizures. CNS Drugs. 2005;19(3):265-272; discussion 273-264.

22. Brodie MJ. Pregabalin as adjunctive therapy for partial seizures. Epilepsia. 2004;45 Suppl 6:19-27.

23. Vera-Llonch M, Brandenburg NA, Oster G. Cost-effectiveness of addon therapy with pregabalin in patients with refractory partial epilepsy. Epilepsia. 2008;49(3):431-437.

24. Gil-Nagel A, Zaccara G, Baldinetti F, Leon T. Add-on treatment with pregabalin for partial seizures with or without generalisation: Pooled data analysis of four randomised placebo-controlled trials. Seizure. 2009;18(3):184-192.

25. Hoppe C, Rademacher M, Hoffmann JM, Schmidt D, Elger CE. Bodyweight gain under pregabalin therapy in epilepsy: mitigation by counseling patients? Seizure. 2008;17(4):327-332.

26. Arain A, Shihabuddin B, Niaz F, et al. Epilepsy and the impact of an epileptology clinic for patients with mental retardation and associated disabilities in an institutional setting. Epilepsia. 2006;47(12): 2052-2057.

27. Modur PN, Milteer WE. Adjunctive pregabalin therapy in mentally retarded, developmentally delayed patients with epilepsy. Epilepsy Behav. 2008;13(3):554-556.
28. Huber B, Bocchicchio M, Feuerbaum E, et al. Efficacy and tolerability of pregabalin in patients with difficult-to-treat epilepsy and intellectual disability. Epilepsy Behav. 2008;13(2):397-401.

29. Seeley JM, Mrelashvili D, Gallagher MJ, Arain A, Abou-Khalil B. Pregabalin efficacy and tolerability in patients with intractable partial seizures. A postmarketing analysis. Epilepsia. 2007;48(s6):320-321.

30. Kubota T, Fang J, Meltzer LT, Krueger JM. Pregabalin enhances nonrapid eye movement sleep. J Pharmacol Exp Ther. 2001;299(3):1095-1105.

31. Romigi A, Izzi F, Marciani MG, et al. Pregabalin as add-on therapy induces REM sleep enhancement in partial epilepsy: a polysomnographic study. Eur J Neurol. 2009;16(1):70-75.

32. Johannessen Landmark C. Antiepileptic drugs in non-epilepsy disorders: relations between mechanisms of action and clinical efficacy. CNS Drugs. 2008;22(1):27-47.

33. Lesser H, Sharma U, LaMoreaux L, Poole RM. Pregabalin relieves symptoms of painful diabetic neuropathy: a randomized controlled trial. Neurology. 2004;63(11):2104-2110.

34. Dworkin RH, Corbin AE, Young JP Jr, et al. Pregabalin for the treatment of postherpetic neuralgia: a randomized, placebo-controlled trial. Neurology. 2003;60(8):1274-1283.

35. Mease PJ, Russell IJ, Arnold LM, et al. A randomized, double-blind, placebo-controlled, phase III trial of pregabalin in the treatment of patients with fibromyalgia. $J$ Rheumatol. 2008;35(3):502-514.

36. Montgomery SA, Tobias K, Zornberg GL, Kasper S, Pande AC. Efficacy and safety of pregabalin in the treatment of generalized anxiety disorder: a 6-week, multicenter, randomized, double-blind, placebocontrolled comparison of pregabalin and venlafaxine. J Clin Psychiatry. 2006;67(5):771-782.

37. Huppertz HJ, Feuerstein TJ, Schulze-Bonhage A. Myoclonus in epilepsy patients with anticonvulsive add-on therapy with pregabalin. Epilepsia. 2001;42(6):790-792.

38. Beydoun A, Nasreddine W, Atweh S. Efficacy and tolerability of pregabalin in partial epilepsy. Expert Rev Neurother. 2008;8(7):1013-1024.

39. Mensah SA, Beavis JM, Thapar AK, Kerr MP. A community study of the presence of anxiety disorder in people with epilepsy. Epilepsy Behav. 2007;11(1):118-124.
Neuropsychiatric Disease and Treatment

\section{Publish your work in this journal}

Neuropsychiatric Disease and Treatment is an international, peerreviewed journal of clinical therapeutics and pharmacology focusing on concise rapid reporting of clinical or pre-clinical studies on a range of neuropsychiatric and neurological disorders. This journal is indexed on PubMed Central, the 'PsycINFO' database and CAS, and is the official

\section{Dovepress}

journal of The International Neuropsychiatric Association (INA). The manuscript management system is completely online and includes a very quick and fair peer-review system, which is all easy to use. Visit http://www.dovepress.com/testimonials.php to read real quotes from published authors. 